\title{
Mitigation of whistling in vertical corrugated pipes by liquid addition
}

\author{
A. C. van Eckeveld $^{1} \cdot$ J. Westerweel ${ }^{1} \cdot$ C. Poelma ${ }^{1}$
}

Received: 20 January 2017 / Revised: 16 June 2017 / Accepted: 26 June 2017 / Published online: 2 August 2017

(C) The Author(s) 2017. This article is an open access publication

\begin{abstract}
When a corrugated pipe is subject to a dry gas flow, high amplitude sound can be produced (so-called 'whistling'). It was shown previously that liquid addition to corrugated pipe flow has the ability to reduce sound production. Small amounts of liquid are sufficient to mitigate whistling entirely. One of the mitigation mechanisms, cavity filling, is studied experimentally. Acoustic measurements are combined with a planar laser-induced fluorescence technique to measure the liquid accumulation in the cavities of a corrugated pipe. Using this technique, it is shown that the amount of filling of the cavities with liquid increases with increasing liquid injection rate and with reducing gas flow rate. The reduction in whistling amplitude caused by the liquid injection is closely related to the cavity filling. This indicates that the geometric alteration of the pipe wall, caused by the accumulation of liquid inside the cavities, is an important factor in the reduction in whistling amplitude.
\end{abstract}

\section{Introduction}

Corrugated pipes are used in many applications throughout industry. These pipes have the advantage of flexibility on the larger (length) scale of the pipes, while still being rigid locally. Typical applications are connections between hard to reach locations and connections that have to allow for movement of the attachment points. When these pipes are subject to an internal gas flow, flow-induced vibrations

A. C. van Eckeveld

a.c.vaneckeveld@tudelft.nl

1 Delft University of Technology, 3ME-PE, Leeghwaterstraat 21, 2628CA Delft, The Netherlands
(FIVs) can occur, caused by an interaction between an acoustic pipe resonance and the unsteady shear layers spanning the corrugations. Under certain conditions, these FIVs result in the production of high-amplitude tonal noise (also known as whistling). This is not only inconvenient, but can lead to damage of equipment, failure of piping systems, and hazardous situations. This study focuses on whistling attenuation by liquid addition to vertical corrugated pipe flow, and the identification of the mechanisms behind this attenuation. For this purpose, a new approach is developed to identify the liquid accumulation within the cavities based on planar laser-induced-fluorescence (PLIF) measurements. These measurements are combined with acoustic measurements to identify the sound production from the corrugated pipes.

Burstyn (1922) and Cermak (1922) were the first of many to study the sound production from single-phase corrugated pipe flow. Since then, many studies have been devoted to the phenomenon behind the whistling behavior, which are summarized in a review paper on corrugated pipe flow by Rajavel and Prasad (2013).

Whistling in corrugated pipes originates from a fluidacoustic feedback. The free shear layers spanning the cavities in this kind of flows are intrinsically unstable and can act as a source of sound. Under certain conditions, discrete vortices can be shed in the cavity mouth. Vortex shedding in the shear layers exerts an unsteady force on the walls, causing a reaction force, which is associated with the sound generation (Curle 1955). This sound source is of a dipole nature due to the vortex-wall interactions (Howe 2003), and feeds an axial acoustic mode in the pipe when the shedding frequency is below the cut-off frequency of the pipe. The acoustic perturbation caused by this acoustic resonance is a source of instability in the shear layers over the corrugations, triggering vortex shedding and closing the feedback loop. 
The onset velocity of whistling was initially thought to be related to a minimum required level of turbulence in the flow (Crawford 1974; Cadwell 1994). Later, it was proposed, based on an energy balance model, that an increase of acoustic losses relative to the source strength at lower flow velocity is a more probable cause for the absence of whistling at low flow speeds (Nakiboğlu et al. 2012b). After onset, a typical discontinuous linear (or "step-wise") increase in whistling frequency is observed, caused by the finite number of possible axial wave modes. The acoustic plane waves, with a frequency below the cut-off frequency of the pipe, are determined by: $f_{n}=n c / 2 L_{\mathrm{p}}$, with $c$ the speed of sound, $L_{\mathrm{p}}$ the pipe length, and $n=1,2,3, \ldots$ Binnie (1961) noticed that the speed of sound in corrugated pipes was slightly different from the speed of sound in free space, and later, Elliott (2005) proposed an effective speed of sound in corrugated pipes:

$c_{\mathrm{eff}}=\frac{c_{0}}{\sqrt{1+V_{\mathrm{c}} /\left(S_{\mathrm{p}} P t\right)}}$,

where $c_{0}$ is the speed of sound in free space, $V_{\mathrm{c}}$ is the cavity volume, $S_{\mathrm{p}}$ the inner cross-sectional area of the pipe, and $P t$ the pitch length (as defined in Fig. 1). Increasing the flow rate after onset of whistling, some of the pipe modes are excited, while others remain silent. This is associated with the acoustic boundary conditions, as shown by Goyder (2010). The linear increase in whistling frequency with flow speed $U$ is associated with a whistling Strouhal number $(S t=f L / U)$. For corrugated pipes, several length scales have been used in the definition of the Strouhal number. Nakiboğlu et al. (2010) showed that: $L=L_{\mathrm{c}}+r_{\text {ed }}$ (see Fig. 1) gave the best collapse of whistling Strouhal numbers for different corrugated pipe geometries.

The effect of corrugation geometry and pitch was first evaluated by Binnie (1961). He observed clear changes in whistling amplitude upon changing the rounding of the rib edges. This was later confirmed by Bruggeman et al. (1991) for side-branch configurations and by Nakiboğlu et al. (2010) for corrugated pipes. Especially rounding the upstream edge of the cavities leads to an increase in whistling amplitude, due to the reduced acoustic absorption

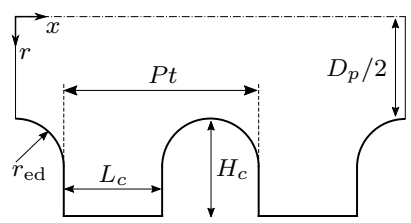

Fig. 1 Schematic of the corrugation geometry and definition of frame of reference near this edge. The authors also looked at the effect of cavity depth on the acoustic source strength (Nakiboğlu et al. 2012a). They demonstrated that above a certain lengthover-depth ratio $\left(L_{\mathrm{c}} / D_{\mathrm{c}} \geq 2\right)$, the source strength significantly decreases when further decreasing the cavity depth. Below this threshold, a very limited effect was noted.

\subsection{Mitigation of whistling}

Several studies were dedicated to mitigation or attenuation of whistling in corrugated pipes. Petrie and Huntley (1980) were among the first to look into whistling mitigation. Their study focused on whistling in vacuum cleaner hoses, and they proposed several ways to reduce sound production from these corrugated pipes. It was observed that obstructions in the flow separation region (for example, using a corrugated pipe with a wrinkled instead of a smooth wall) significantly reduce the whistling amplitude. They also found that the incoming boundary layer thickness is of major importance to the occurrence of whistling, since it determines whether or not the shear layers spanning the corrugations become unstable. Attempts that made use of active noise control with small speakers at a single corrugation did not prove to be very successful (Gharib 1993) and are not feasible in many industrial applications. More recently, Rudenko et al. (2013) came up with a semi-empirical model for corrugated pipes with smooth segments. With the acoustic amplitude obtained from two-dimensional axisymmetric URANS simulations, the model determines the smooth pipe length required to damp the acoustic waves generated by the corrugated segment, essentially by increasing the onset velocity to higher values, compared to the fully corrugated pipe.

Addition of liquid to the gas flow through a corrugated pipe has the potential to mitigate whistling entirely; see Belfroid et al. 2013, 2014; Golliard et al. 2013. These studies focused on sound mitigation in horizontal pipes, with some experiments in a vertical orientation, and a field case study. Small liquid fractions were sufficient to prevent whistling in most cases. The authors proposed several whistling mitigation mechanisms: filling of the cavities with liquid; additional acoustic damping; and a reduction of the acoustic source strength due to boundary layer thickening. As a follow-up, the authors conducted experiments in side-branch configurations with liquid addition (Sanna et al. 2015). In those configurations, they found that whistling mitigation is mainly caused by the additional acoustic damping caused by the presence of liquid. Therefore, the flow regime plays an important role, especially for the horizontal pipes they studied. The relevance of the different mechanisms behind mitigation of whistling in corrugated pipes is still unclear. 


\subsection{Dimensionless parameters}

Several dimensionless parameters are used in the current work and their definition is given here. Figure 1 gives an overview of the geometric parameters concerning the corrugated pipe. The used geometry is a simplified version of commonly used corrugated pipes. For single-phase whistling, two parameters are of particular importance; these are the Mach and the Reynolds numbers. Normalization of the bulk velocity $\left(U_{\mathrm{b}}\right)$ with the speed of sound $\left(c_{0}\right)$ gives the Mach number:

$M a=U_{\mathrm{b}} / c_{0}$.

The flow in this work is considered to be incompressible $\left(M a^{2} \ll 1\right)$. The whistling frequency $f_{\mathrm{w}}$ is normalized using the Strouhal number, where the length scale proposed by Nakiboğlu et al. (2010) is applied:

$S t=f_{\mathrm{w}}\left(L_{\mathrm{c}}+r_{\mathrm{ed}}\right) / U_{\mathrm{b}}$.

Also the Reynolds number is important, which compares inertial and viscous forces in the gaseous bulk flow: $R e$ $=\rho_{\mathrm{g}} U_{\mathrm{b}} D_{\mathrm{p}} / \mu_{\mathrm{g}}$, with $\rho_{\mathrm{g}}$ and $\mu_{\mathrm{g}}$ being the mass density and the dynamic viscosity of the gas phase, respectively.

For two-phase flow through corrugated pipes, a couple of additional dimensionless numbers are relevant: the Weber number compares the inertial forces (IN) against the surface tension (SF): We = IN/SF; the Bond number compares the gravitational forces (GR) against surface tension: $B o=\mathrm{GR} / \mathrm{SF}$. For the specific situation studied in this work, these forces are defined as:

$\mathrm{IN}=\rho_{\mathrm{g}} U_{j}^{2} L_{j} L_{\mathrm{r}}, \quad \mathrm{GR}=\Delta \rho \mathrm{g} D_{\mathrm{c}} L_{\mathrm{c}} L_{\mathrm{r}} \alpha, \quad \mathrm{SF}=\sigma L_{\mathrm{c}}$.

$\alpha$ is the fraction of the cavity volume occupied by liquid. The accompanying length scales and velocities are estimated based on experimental work on cavities in a flat plate (Koschatzky et al. 2011) and are defined as

$L_{j}=L_{\mathrm{c}}^{*} / 4, \quad L_{\mathrm{r}}=\pi D_{\mathrm{p}}, \quad U_{j}=U_{\mathrm{b}} / 4$.

The jet-like flow directed into the cavity over the downstream cavity wall is the main source of inertial forces exerted at the liquid inside the cavity. The average velocity in this jet is taken as $25 \%$ of the bulk velocity, which is a typical value found for flat plate cavities. The streamwise length scale for this jet is taken as one quarter of the free cavity length $L_{\mathrm{c}}^{*}$, which is defined in Sect. 4.2.1. The curvature of the surface, used in the definition of the surface tension forces, is the cavity length. Table 1 gives the range for the Bond and Weber numbers attained in the current experiments. Surface tension is in all cases negligible compared to gravitational and inertial forces.
Table 1 Range of dimensionless parameters attained in the present experiments

\begin{tabular}{ll}
\hline Quantity & Range \\
\hline$R e_{\mathrm{g}}$ & $10^{4}-10^{5}$ \\
We & $10^{1}-10^{2}$ \\
Bo & $10^{1}-10^{2}$ \\
\hline
\end{tabular}

\subsection{Objective and outline}

This study focuses on the effect of liquid addition to whistling in upward gas flow through vertical corrugated pipes. As stated in the previous section, the relevance of the different mechanisms behind whistling mitigation by liquid addition remains a subject of further study. This work intends to contribute to answering this question by investigating specifically the influence of filling of the corrugations to whistling mitigation. For that purpose, an experimental setup was developed aimed at measuring the liquid accumulation within the corrugations while simultaneously measuring the acoustic output of the corrugated pipe. In Sect. 2, an overview of the experimental setup is provided, followed by the measurement procedure and data processing in Sect. 3. The results are presented in Sect. 4, followed by a discussion in Sect. 5. Section 6 provides conclusions from this work and an outlook to future work.

\section{Experimental setup}

The measurements were carried out in a dedicated experimental setup with a vertical test section. A schematic representation of the setup is depicted in Fig. 2. Air flow through the pipe is provided by a blower (Esam Mediojet $2 \mathrm{~V}$, A in the figure). A long-radius ASME flow nozzle, having a zero $\beta$ ratio (Leutheusser 1964), is used as a flow measuring device. Comparison of the flow rate obtained using this nozzle, with pitot tube measurements, yielded a maximum error in the volumetric flow rate that is smaller than $4 \%$ for all cases. The blower is connected to an expansion vessel, with a diameter of $0.5 \mathrm{~m}$ and a length of $1 \mathrm{~m}$ (B in Fig. 2). The vessel is covered on the inside with a $10-\mathrm{cm}-$ thick layer of sound absorbing foam to reduce acoustic resonances in the interior of the vessel. The expansion vessel both serves as a damper for noise generated upstream by the blower, and it provides a high acoustic reflection coefficient boundary condition upstream of the corrugated pipe. Together with the high acoustic reflection at the open outflow of the pipe, it enhances whistling by strengthening the acoustic standing wave inside the corrugated pipe. The acoustic measurement section (C in Fig. 2) consists of four 


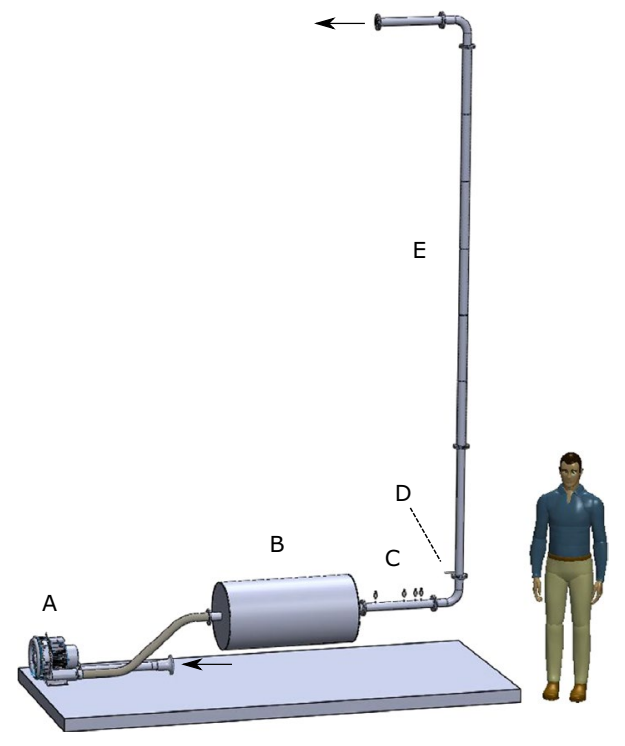

Fig. 2 Schematic representation of the experimental setup. $A$ blower, $B$ expansion vessel, $C$ acoustic measurement section, $D$ liquid injection point, and $E$ corrugated section. A more detailed description is found in the text

microphones (PCB 106B) flush mounted in the pipe wall. The positions of the microphones were chosen to ensure a truthful reconstruction of the acoustic waves in the frequency range of interest $(0-3.5 \mathrm{kHz})$. Following the work by Jang and Ih (1998), the second, third, and fourth microphones were placed at distances to the first microphone of $0.225,0.315$, and $0.360 \mathrm{~m}$, respectively. The microphones are connected to a data acquisition card (NI PCI-4472). Dynamic pressure signals obtained from the acoustic measurement section were used to reconstruct the traveling waves in the main pipe, from which the standing acoustic wave is obtained (as explained in Sect. 3.1). A pressure tap is placed just before the liquid injection point (D), to make sure that the presence of liquid does not interfere with the pressure measurements. The static pressure at this point is used to correct the flow rate for changes in density. Liquid is injected into the pipe between the pressure tap and the corrugated pipe section, using spray nozzles (Bete PJ8, PJ10, PJ15, and PJ24) connected to a rotary vane pump (Fluid-O-Tech PA111). The nozzles produced a dispersed spray of droplets with a nominal diameter less than $150 \mu \mathrm{m}$.

\footnotetext{
1 An estimation of the evaporation rate is done based on the Sherwood number (Froessling 1938), assuming that all liquid is present as $100-\mu \mathrm{m}$ size droplets being transported with the gas velocity. The evaporation will be in the order of $5 \%$ in the range of interest. The actual evaporation rate is expected to be lower, since the evaporation from liquid present in the film at the pipe wall is considerably lower compared to droplet evaporation. The liquid flow rate is, therefore, not corrected for evaporation.
}

The liquid flow rate was measured using a Coriolis mass flow meter (Bronkhorst M14 CORI-FLOW). ${ }^{1}$ For flow development purposes, a 1-m-long smooth pipe section $\left(L / D_{\mathrm{p}}=20\right)$ is placed behind the liquid injection point, followed by the corrugated pipe section $(E)$. The flow loop is terminated with an open outflow, blowing into a large room $\left(5 \times 10 \times 4 \mathrm{~m}^{3}\right)$, without any acoustical treatment of the walls. Temperature measurements are performed at the outlet using a Pt100 temperature probe located at the pipe center-line. The temperature measurements are used to correct the mass density of the gas and the speed of sound (assuming an ideal gas: $c_{0}=\sqrt{\gamma \mathrm{RT}_{\text {air }} / M_{\text {air }}}$, where $\gamma$ is the ratio of specific heats, $R$ the ideal gas constant, $T_{\text {air }}$ the temperature inside the pipe, and $M_{\text {air }}$ the molar mass of air). The speed of sound has not been corrected for the presence of droplets in the gas phase. At the very low volume fractions of liquid in suspension reported in this study, the effect on the speed of sound is rather limited (Kieffer 1977).

The smooth piping is made of steel, with an inner diameter $\left(D_{\mathrm{p}}\right)$ of $49.25 \mathrm{~mm}$, and a wall thickness of $5 \mathrm{~mm}$. This high wall thickness was chosen to prevent structural vibrations that might influence the measurements. The corrugated section was machined from PVC and has a minimum wall thickness of $10 \mathrm{~mm}$. One particular corrugation geometry is used in this study, schematically depicted in Fig. 1. The depth $\left(D_{\mathrm{c}}\right)$ and length $\left(L_{\mathrm{c}}\right)$ of the corrugations are $4 \mathrm{~mm}$, with rounded upstream and downstream edges, both having a radius $\left(r_{\text {ed }}\right)$ of $2 \mathrm{~mm}$. The edges were rounded, because this was shown to enhance the whistling behavior of corrugated pipes (Nakiboğlu et al. 2010). The pitch length $(P t)$ is $8 \mathrm{~mm}$ resulting in a zero plateau length between the ribs, which reduces the onset velocity for whistling of the corrugated pipe. The dimensions were chosen to obtain a large Mach number range resulting in whistling in the pipe. The length of the corrugated pipe could be varied, but was maintained constant during the reported measurements, with a length of $3 \mathrm{~m}\left(L_{\mathrm{p}} / D_{\mathrm{p}} \approx 61\right)$, containing 375 corrugations. Increasing the corrugated pipe length further is not expected to add to the whistling amplitude (Nakiboğlu et al. 2011).

For optical access, a transparent corrugated section was placed just before the end of the corrugated pipe, at $49 \leq L / D \leq 53$ from the corrugated pipe entrance. Although an improved refractive index matching could be achieved using a different material (e.g., FEP), the section was made from PMMA, since it is easier to machine a corrugated pipe from this material. A planar laser-inducedfluorescence (PLIF) technique was used to measure the liquid accumulation in the corrugations. The experimental layout of the PLIF measurement setup is shown in Fig. 3. The transparent section is contained in an optical box made from PMMA to reduce image distortion due to 


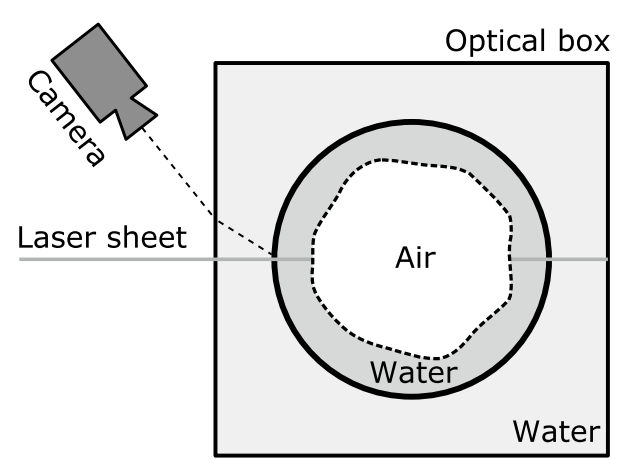

Fig. 3 Schematic of the PLIF setup used in the visualization measurements, seen from the top. The water at the interior of the pipe is dyed with $125 \mu \mathrm{g} / \mathrm{L}$ rhodamine, water in the optical box with $50 \mu \mathrm{g} / \mathrm{L}$

refraction of light at the curved outer pipe wall. The optical box was filled with water. To reduce the effect of total internal reflection at the liquid-gas interface inside the pipe and the effect of refraction at the water-PMMA interface at the pipe wall, the camera was put at a viewing angle of $25^{\circ}$ with respect to the laser sheet. With a steady and perfectly axisymmetric gas-liquid interface within the corrugations, this yields a viewing angle well below the critical angle for air-water $\left(=48.6^{\circ}\right)$. A digital camera $(\mathrm{LaVi}$ sion Imager Intense, 1MP sensor) was used, equipped with a $105 \mathrm{~mm}$ focal length (Nikkor) objective and a red filter (B + W 62 041) to capture only the fluorescent light from the fluorescent dye (Rhodamine WT) that was added to the injected water. The dye concentration was optimized for the required light intensity of the emitted fluorescent light, resulting in a concentration $125 \mu \mathrm{g} / \mathrm{L}$ water. Furthermore, the optical box was filled with a Rhodamine solution of approximately $50 \mu \mathrm{g} / \mathrm{L}$, and the fluorescent light emitted from this region was used to correct for non-uniformity in the laser sheet.

\section{Measurement procedure and data processing}

\subsection{Acoustic measurements}

Acoustic pressure measurements were acquired at a sampling frequency of $40 \mathrm{kHz}$, which is well above the cut-off frequency of the pipe to ensure that aliasing effects would not interfere with the sampling rate. Time series were collected with a duration of $1 \mathrm{~s}$. For each set of parameters, the measurements were repeated four times and checked for consistency. The dynamic pressure signals were acquired simultaneously with static pressure, temperature, and flow rate measurements to compute the actual flow rate through the corrugated section.
The acoustic waves in the pipe were reconstructed using the multiple microphone method (Jang and Ih 1998). Since the whistling frequencies are always below the cut-off frequency of the pipe, the acoustic field is composed of plane waves traveling back and forth through the pipe. The acoustic field can then be described by

$P(x)=P^{+} \exp \left(-i k^{+} x\right)+P^{-} \exp \left(i k^{-} x\right)$,

where $P$ is the complex-valued amplitude of the dynamic pressure $\left(p(x, t)=P(x) e^{i \omega t}\right)$ at position $x, P^{+}$and $P^{-}$are the amplitudes of the upstream and downstream traveling acoustic waves, and $k^{+}$and $k^{-}$the respective complex wavenumbers. Measuring the dynamic pressure at $n$ positions along the pipe results in the following overdetermined linear system of equations that can be solved for $P^{+}$and $P^{-}$ using a least-squares approach:

$\left[\begin{array}{cc}\exp \left(-i k^{+} x_{1}\right) & \exp \left(i k^{-} x_{1}\right) \\ \exp \left(-i k^{+} x_{2}\right) & \exp \left(i k^{-} x_{2}\right) \\ \vdots & \vdots \\ \exp \left(-i k^{+} x_{n}\right) & \exp \left(i k^{-} x_{n}\right)\end{array}\right]\left(\begin{array}{c}P^{+} \\ P^{-}\end{array}\right)=\left(\begin{array}{c}P_{x_{1}} \\ P_{x_{2}} \\ \vdots \\ P_{x_{n}}\end{array}\right)$.

Neglecting the acoustic attenuation by turbulent fluctuations in the bulk of the flow (since the ratio of acoustic boundary layer thickness-to-thickness of the viscous sublayer of the turbulent mean flow is in the order of one: $\delta_{a c}^{+}=\delta_{a c} / \delta_{l} \approx 1$; see Weng 2015), the complex wavenumbers are found using the model proposed by Dokumaci (1995). Assuming a uniform mean velocity profile in the pipe, he derived the following expression for the wavenumber, valid for large shear numbers $\left(S h=0.5 D_{\mathrm{p}} \sqrt{\omega / v} \gg 1\right)$ :

$k^{ \pm}=k_{0} \frac{ \pm \Gamma_{0}}{1 \pm \Gamma_{0} M a}$.

In this equation, $k_{0}$ is the wavenumber in free space $\left(k_{0}=\omega / c_{0}\right)$ and $\Gamma_{0}$ the dimensionless wavenumber for sound propagation through a quiescent fluid inside a pipe, taking into account the viscothermal damping only. At high shear numbers, $\Gamma_{0}$ can be approximated by (Ronneberger and Ahrens 1977):

$\Gamma_{0}=1+\frac{1-i}{\operatorname{Sh} \sqrt{2}}\left(1+\frac{\gamma-1}{\sqrt{P r}}\right)-\frac{i}{S h^{2}}\left(1+\frac{\gamma-1}{\sqrt{P r}}-\gamma \frac{\gamma-1}{2 P r}\right)$,

where $P r$ is the Prandtl number, and $\gamma$ the ratio of specific heats. Considering a high acoustic reflection coefficient $(R)$ at both pipe ends, a standing wave is present in the pipe, with a maximum amplitude of $P_{\mathrm{st}}=P^{+}+P^{-}$. The reflection coefficients at both pipe ends were determined in a dedicated setup, using the multiple microphone method 


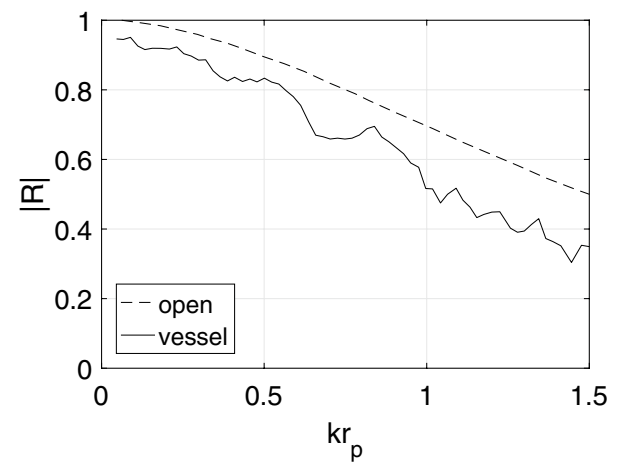

Fig. 4 Absolute value of the acoustic pressure reflection coefficient for the upstream expansion vessel as a function of $k r_{\mathrm{p}}(-)$, compared to the reflection coefficient for an unflanged open pipe end termination (- -), based on Levine and Schwinger (1948). $k r_{\mathrm{p}}=\frac{1}{2} k D_{\mathrm{p}}$, is the wavenumber, non-dimensionalized with the pipe radius

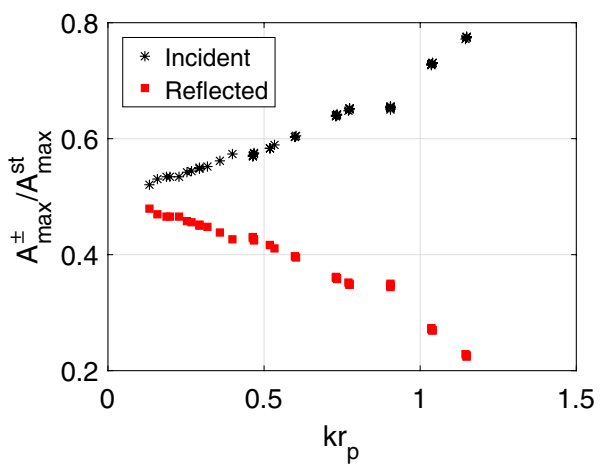

Fig. 5 Typical measured amplitude of the incident and reflected acoustic waves at the expansion vessel versus $k r_{\mathrm{p}}$. The wave amplitude was made dimensionless with the maximum whistling amplitude observed in the respective standing wave. Stronger deviation at higher $k r_{\mathrm{p}}$ is due to the reduced reflection coefficient at higher frequencies

(similar to Peerlings 2015). The reflection coefficient is defined as the ratio of the incoming and reflected wave amplitude at the pipe end: $R_{x_{0}}=P_{x_{0}}^{-} / P_{x_{0}}^{+}$. It was found that the reflection coefficient for the upstream and downstream acoustic termination is not equal to one. Especially for higher values of $k r_{\mathrm{p}}$ (where the wavenumber $k$ is nondimensionalized with the pipe radius: $r_{\mathrm{p}}=\frac{1}{2} D_{\mathrm{p}}$ ), it tends to deviate (Fig. 4). This causes the acoustic waves traveling towards the expansion vessel to be significantly higher in amplitude compared to the reflected waves traveling in the opposite direction (Fig. 5). The main region of interest for the present study is located towards $k r_{\mathrm{p}}=1$, where $|R|$ attains values below 0.7 . There will, therefore, be a footprint of the acoustic boundary condition on the whistling amplitude. A standing wave is, however, still observed in

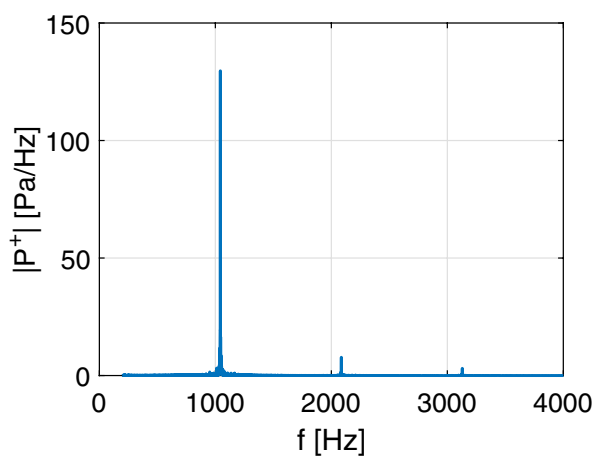

Fig. 6 Typical frequency spectrum obtained from the acoustic pressure measurements, at $M a=0.058\left(U_{\mathrm{b}}=20 \mathrm{~m} / \mathrm{s}\right)$

the range of interest, and the amplitude of this standing wave was used as a representation of the strength of the acoustic field generated in the corrugated pipe $\left(A_{\max }^{\text {st }}\right)$. A typical spectrum, obtained using the described method, is depicted in Fig. 6.

Whistling was identified using the whistling criterium proposed by Nakiboğlu et al. (2010). For every data set, the frequency with the highest amplitude of whistling $\left(f_{\mathrm{w}}\right)$ was identified, and the energy $\left(E_{\mathrm{w}}\right)$ contained within \pm 1 $\mathrm{Hz}$ of that frequency is compared to the energy $\left(E_{\text {sig }}\right)$ in the entire discrete Fourier transform (DFT) of the time series. This bandwidth was used, because it contains most of the energy in the peak in the frequency spectrum, as is clear from Fig. 6. The ratio between the two was used as a criterium for whistling to occur, i.e., whistling occurs when $W>0.1$, with:

$W=\frac{E_{\mathrm{w}}}{E_{\text {sig }}}$.

Subsequently, a sine wave was fitted to the raw signal with the same whistling frequency $\left(f_{\mathrm{w}}\right)$. The whistling amplitude $\left(A_{\mathrm{w}}\right)$ was obtained by adapting the amplitude of the sine wave, so that its energy content matches $E_{\mathrm{w}}$. To evaluate this procedure, the repeatability of the whistling amplitude and frequency was tested, and was found to be within $1 \%$, when keeping the flow settings constant.

\subsection{PLIF measurements}

PLIF measurements are used for quantification of the amount of liquid that accumulated inside the corrugations. Images are recorded at a frame rate of $2 \mathrm{~Hz}$, with each series containing 200 images and repeated twice for every measurement point within the parameter space of interest. First, the required gas flow rate was set, and liquid injection was switched on, starting from the highest 
liquid flow rate achievable with the nozzle in place. After ensuring full wetting of the interior of the pipe, the liquid flow rate was adjusted to the desired value. Data acquisition was only started when the acoustic output and the average filling had reached an equilibrium state. During all PLIF measurements, two series of acoustic measurements were acquired, following the procedure described in Sect. 3.1.

To get from the raw images to a measure of the liquid accumulation within the cavities, some image processing steps were applied. The different steps are illustrated in Fig. 7, starting from the raw image on top (Fig. 7a) and resulting in the detected liquid regions in the bottom image (Fig. 7f). Eight cavities are in view in the images, where flow is from left to right. The raw image (Fig. 7a) shows the large high-intensity region below the corrugations, which is caused by the liquid inside the optical box (as described in Sect. 2). The intensity distribution of the laser sheet is obtained from the captured light intensity from this region.
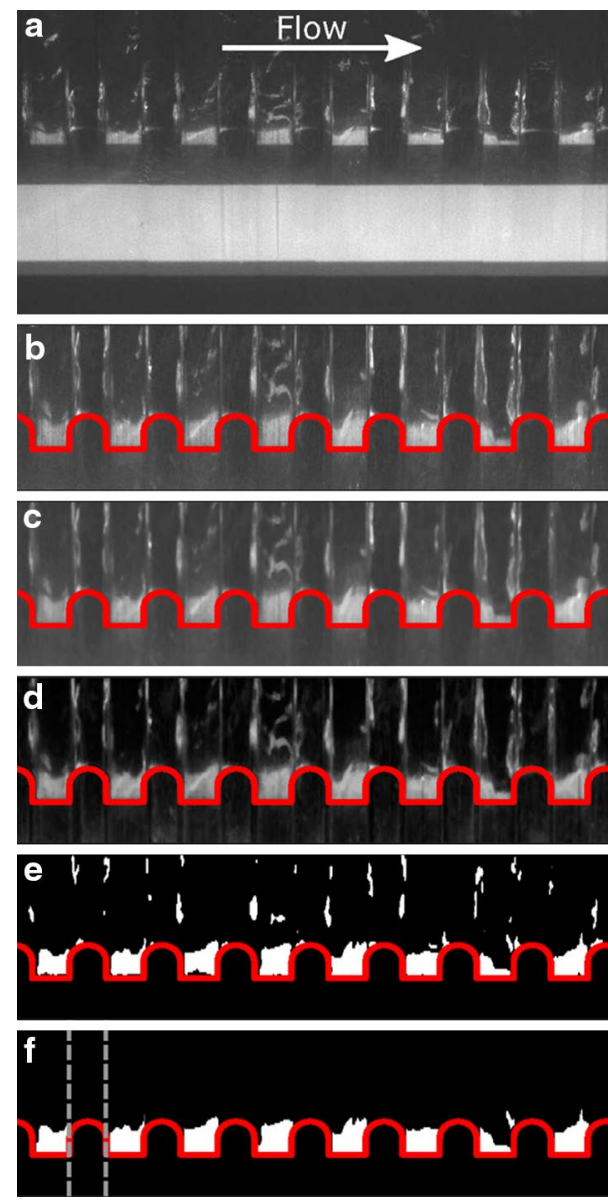

Fig. 7 Steps taken in processing the recorded PLIF images, illustrated for the case with downward oriented flow $\left(U_{\mathrm{b}}=35 \mathrm{~m} / \mathrm{s}\right.$ and $Q_{1}=305 \mathrm{~mL} / \mathrm{min}$ ). Flow is from left to right. The internal corrugated pipe wall is drawn in red for the reconstructed images. The image processing steps are explained in the text
It is subsequently used to correct the measured gray values in the images, in streamwise direction. The image is subsequently corrected for the distortion caused by the $25^{\circ}$ angle of view with respect to the laser sheet (Fig. 7b). A moving average filter is applied (filter size $3 \times 1$ pixels) to reduce artefacts caused by wall imperfections (e.g., scratches on the surface). A $3 \times 3$ pixel median filter is applied to further reduce single-pixel noise in the images. The result is shown in Fig. 7c. The contrast is enhanced using row-byrow stretching of the gray level $(I)$ in the radial direction (Fig. 7d), using:

$I_{\mathrm{s}}=\frac{I-\min (I)}{\max (I)}$.

Subsequently a straightforward binarization step is applied (resulting in Fig. 7e). Gaps between liquid filling and the rib interior are filled, and liquid regions that are entirely disconnected from the pipe wall are omitted. Furthermore, only the liquid detected within the corrugations is preserved, because the regions above the curved ribs (between the dashed gray lines in Fig. 7f) are distorted by the light refraction at the pipe wall liquid interface. The image processing steps result in a binary filling image (Fig. 7f), where liquid only occurs between the ribs.

Some of the shortcomings of using a PLIF-based technique for the detection of liquid accumulation in a pipe flow can be observed from Fig. 7. First of all, the pipe wall is machined and polished afterwards. This, however, always leaves some surface defects, especially in the bottom corners of the cavities, that affect the transparency of the pipe. Apart from wall defects, total internal reflection at the gas-liquid interface also introduces errors in the amount of liquid detected. Although a viewing angle was used that is well below the critical angle for a steady and flat interface, total reflection still occurs when the surface is inclined with respect to the pipe wall or when surface waves are induced by the gas flow over the interface. This results in an overestimation of the thickness of the liquid film close to the wall (up to a factor of two for steep surface angles in smooth walled annular pipe flow and a viewing angle of $90^{\circ}$; see Häber et al. 2015).

Nevertheless, the PLIF-based technique is useful to observe trends in the filling behavior and to evaluate the liquid accumulation for different flow parameters.

\section{Results and discussion}

\subsection{Single-phase whistling}

Single-phase experiments were carried out to characterize the experimental setup and are used as a reference to 


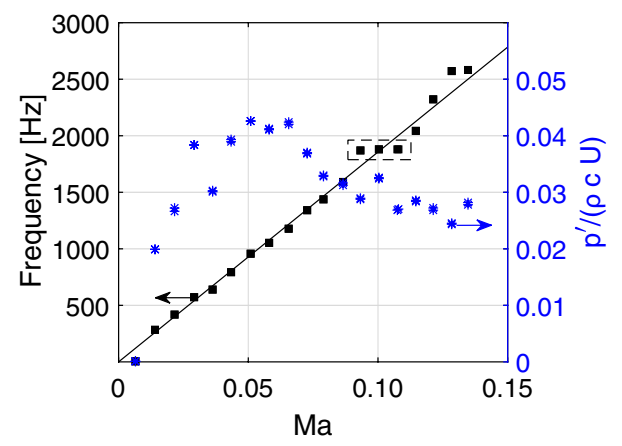

Fig. 8 Dry whistling over the entire Mach number $(M a)$ range, with upward directed gas flow and the injection nozzle installed. Whistling frequency is displayed by and whistling amplitudes by $*$. The solid line indicates $S t=0.32$. Dashed box gives position of data presented in Fig. 10

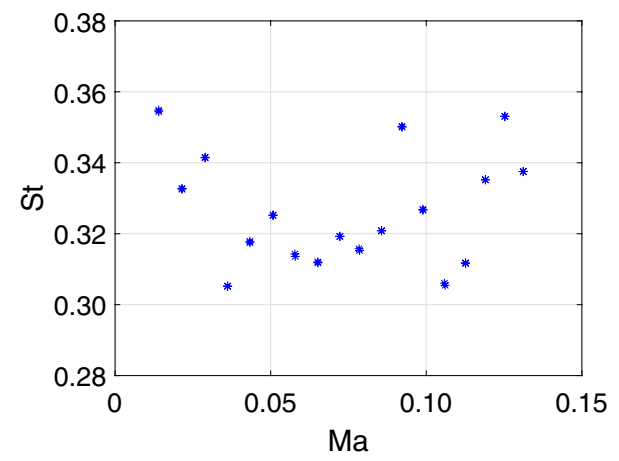

Fig. 9 Strouhal $(S t)$ vs. Mach $(M a)$ number plot for dry whistling in the corrugated pipe

the two-phase flow study presented in the subsequent section. Figure 8 shows the whistling behavior of the corrugated pipe with upward directed gas flow. The whistling frequency linearly increases with increasing Mach number, indicating a fixed whistling Strouhal number $(S t)$. Using $L_{\mathrm{c}}+r_{\text {ed }}$ as the characteristic length scale (Nakiboğlu et al. 2010) gives a whistling Strouhal number of approximately 0.32 , as shown in Fig. 9. The discontinuous increase in whistling frequency indicates a strong lock-in between axial acoustic pipe modes and the vortex shedding within the corrugations, manifested by plateaus of constant whistling frequency. This also causes the scatter in the whistling Strouhal number as observed in Fig. 9. The axial acoustic modes that are excited range up to a mode number $\left(n_{\mathrm{a}}=2 L_{\mathrm{t}} f / c_{\text {eff }}\right)$ of 86 , where $L_{\mathrm{t}}$ is the total pipe length. The dimensionless whistling amplitude strongly increases from the onset velocity up to a Mach number of 0.025 , after which it stabilizes. For higher Mach numbers $(M a>0.075)$ a gradual decrease in whistling amplitude is observed. This is caused by the reduced acoustic reflection of the

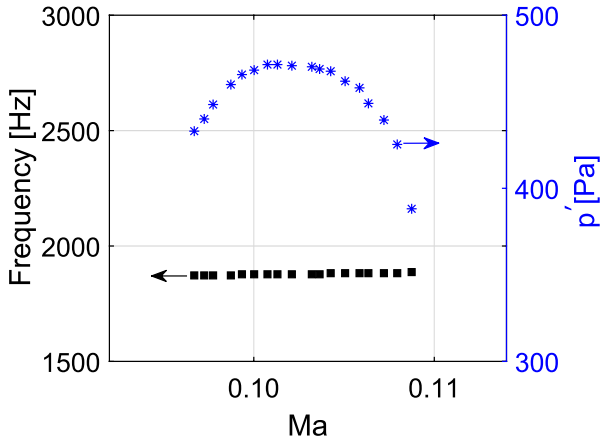

Fig. 10 Dry whistling amplitude and frequency as a function of Mach number $(M a)$ at a single frequency plateau. Whistling frequency is displayed by and whistling amplitudes by $*$. Parameter range is indicated in Fig. 8 with a dashed rectangle

terminations at the upstream and downstream ends of the pipe, as discussed in Sect. 3.1.

Large variations in whistling amplitude occur in Fig. 8. The whistling amplitude within a single plateau peaks in the middle decreases towards the beginning and the end of the plateau (as shown in Fig. 10). Due to the coexistence of multiple whistling frequencies at the edges of the plateaus, the total acoustic energy is divided over a larger frequency range, yielding a lower whistling amplitude at a single frequency. Since the Mach number in the dry whistling experiments was gradually increased, whistling was not always recorded at the maximum whistling amplitude occurring at a certain frequency. Dips in the whistling amplitude largely coincide with points close to jumps in whistling frequency, where both modes appear simultaneously in the frequency spectrum. For the evaluation of the attenuation of whistling by the presence of a liquid phase (described in the next section), this is not problematic, since the acoustic amplitude is always compared to the amplitude at dry whistling conditions at the same Mach number.

\subsection{Whistling attenuation by a liquid phase}

\subsubsection{Upward gas flow}

The injection of small amounts of liquid into the core of the gaseous flow results in a clear decrease in whistling amplitude for upward oriented gas flows (Fig. 11), similar to what was found by Belfroid et al. (2013). At the lowest gas flow rate, where $M a=0.1\left(U_{\mathrm{b}}=35 \mathrm{~m} / \mathrm{s}\right)$, the strongest effect is observed. A liquid volume fraction is defined, based on the gas and liquid fluxes: $\Phi_{1}=\frac{Q_{1}}{Q_{1}+Q_{\mathrm{g}}}$, where $Q_{1}$ is the injected liquid flux and $Q_{\mathrm{g}}$ the gas flux. A fraction of $2 \times 10^{-5}$ was already sufficient to prevent the pipe from whistling. Higher gas flow rates required higher volume fractions of liquid to be added to the gas flow, to mitigate 


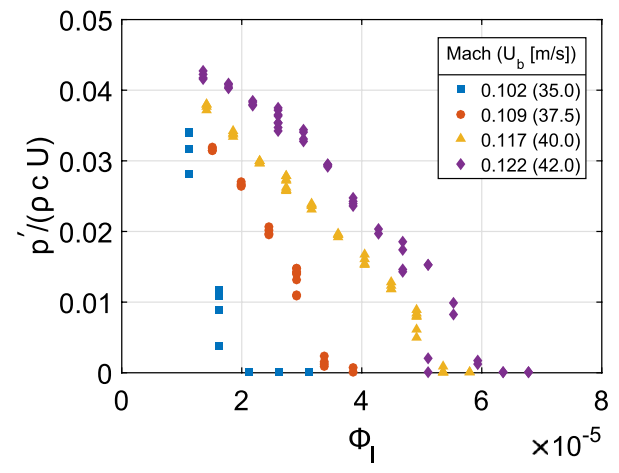

Fig. 11 Dimensionless whistling amplitude plotted against the volume fraction of liquid injected into the corrugated pipe flow, at different Mach numbers

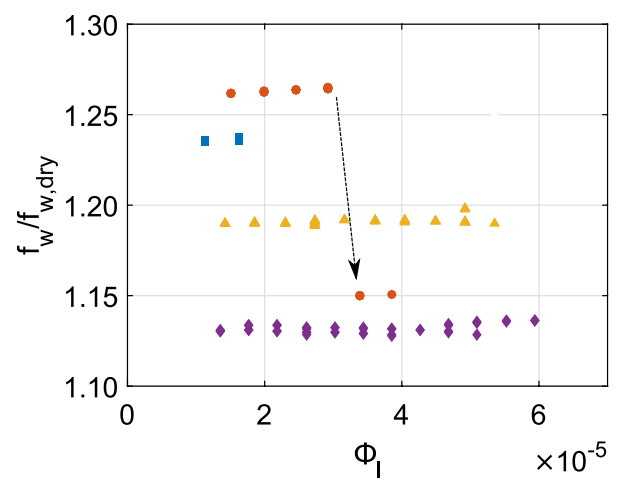

Fig. 12 Change in whistling frequency relative to the dry whistling case as a result of liquid injection. Largest jump is observed for $M a=$ $0.109\left(U_{\mathrm{b}}=37.5 \mathrm{~m} / \mathrm{s}\right)$, and is indicated with the arrow. Symbols are as defined in Fig. 11

whistling entirely. A nearly linear relation was found between the volume fraction of water added and the dimensionless acoustic amplitude of whistling.

Not only the whistling amplitude is affected by liquid injection, also the frequency changes. This change is, however, not as drastic. The whistling frequency increases by $12 \%$ to over $25 \%$ when liquid is injected (Fig. 12). Two effects could invoke this increased frequency. First, assuming a constant whistling Strouhal number, a decreased cavity length leads to an increase in frequency. However, since the frequency does not change significantly upon increasing the liquid volume fraction in the flow further, the relation with the reduced cavity length does not seem to hold. Since mainly the length of the cavity mouth $\left(L_{\mathrm{c}}+r_{\mathrm{ed}}\right)$ is important for the whistling frequency, only liquid accumulating in the cavity mouth influences this parameter. The filling of the corrugations, however, starts from the bottom corner and progresses upward, causing the length of the cavity mouth to be fairly constant over a large range of liquid injection rates (see also Fig. 16, which is to be discussed later). The second effect is the changed rib geometry by liquid flowing over the ribs. This occurs in all cases where liquid is added to the corrugated pipe flow. Since the upstream and downstream edge geometry is of major importance to the whistling characteristics, geometrical alterations of these rib edges are expected to affect the whistling process significantly (Nakiboğlu et al. 2010).

The sudden reduction of whistling frequency for higher liquid injection rates, for $M a=0.109\left(U_{\mathrm{b}}=37.5\right.$ $\mathrm{m} / \mathrm{s}$ ), is caused by a jump from one plateau to the other. At this flow speed, whistling is very close to the edge of a plateau, resulting in a double peaked frequency spectrum. Both peaks represent two neighboring plateaus. Whistling is less stable here and switches between two plateaus at higher liquid volume fractions. This process is stimulated not only by the altered corrugation geometry, but also by an increase in the effective speed of sound due to a reduction of the cavity volume (Eq. 1).

The PLIF images are used to obtain a measure for the cavity filling by the liquid phase. After the image processing steps (described in Sect. 3.2), the filling of the individual corrugations was averaged over all images in a measurement series and over all ribs in the field of view, yielding a spatially averaged and time-averaged filling of a cavity. Figure 13 shows a typical measured average filling profile, here for $M a=0.117(U=40 \mathrm{~m} / \mathrm{s})$ and $\phi_{\mathrm{L}}=1.85 \times 10^{-5}\left(Q_{1}=85 \mathrm{~mL} / \mathrm{min}\right)$. The flow is from left to right and gravity points in opposite direction. Liquid is blown away from the downstream cavity edge due to the inertial force exerted by the incoming gas flow and due to gravity, dragging the heavier liquid towards the upstream cavity edge. The recirculating gas flow inside the cavity pushes liquid back towards the shear layer origin, where it is re-entrained in the gaseous core flow in the pipe. A more detailed analysis of these forces is given in Sect. 5. Small

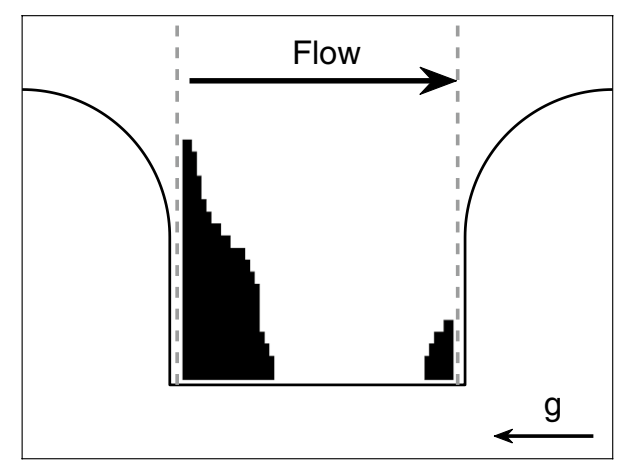

Fig. 13 Averaged cavity filling obtained from PLIF imaging. Flow is from left to right, $M a=0.117(U=40 \mathrm{~m} / \mathrm{s})$ and $\phi_{\mathrm{L}}=1.85 \times 10^{-5}$ $\left(Q_{1}=85 \mathrm{~mL} / \mathrm{min}\right)$. Dark regions are regions of liquid accumulation and the area outside dashed lines has been masked 


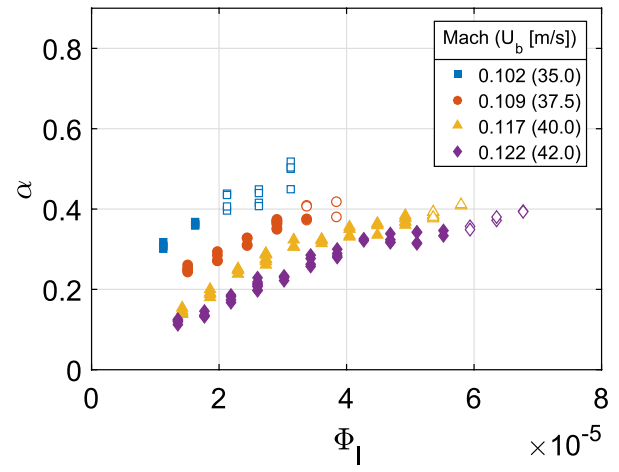

Fig. 14 Time and space-averaged filling of corrugations for increasing liquid flow rate. Closed symbols represent the whistling cases, whereas open symbols are the non-whistling cases

amounts of liquid are also found at the bottom downstream corner of the cavities, kept there by surface tension forces. Note that the liquid that is topping the ribs is not visible in the processed images. There is, however, liquid flowing over the ribs along the pipe wall, as can be seen in Fig. 7.

The fill fraction $(\alpha)$ of the averaged images was calculated, and the results are plotted against $\Phi_{1}$ in Fig. 14. There is a clear increase in filling with increasing liquid flow rate, which was not observed before. This indicates that there is a dynamic equilibrium, where the liquid content of the boundary layer approaching the corrugations plays an important role. There is a continuous refreshment of the liquid inside the cavities. When switching back to injection of fresh water, it took several minutes before the rhodamine solution was entirely replaced. Below a mean cavity filling of 30\%, a linear increase with $\Phi_{1}$ is observed. At higher filling rates, the trend departs from this linear behavior, and a slower increase in filling is obtained when further increasing the liquid volume fraction, especially at higher gas flow speeds. The gas flow rate has a large effect on $\alpha$. A decrease in flow rate of $16 \%$ results in more than a doubling in $\alpha$.

The open markers in Fig. 14 represent non-whistling cases. From the figure, it follows that above a fixed filling threshold ( $\alpha \approx 0.38$ ), no whistling is observed, independent of the gas flow rate. In all cases, the whistling stops ( $W$ drops below 0.1 ) when corrugations are filled above this threshold. This indicates that there is a critical filling above which whistling entirely disappears. A more detailed evaluation of this trend is found by plotting the whistling amplitude versus the average rib filling (Fig. 15). All data then closely collapse onto a single curve, showing the decreasing acoustic amplitude for increasing liquid accumulation within the corrugations. The whistling amplitude steadily decreases from 10 to $30 \%$ filling, after which a stronger decline in sound production is observed upon further increasing the filling. The strong correlation between liquid accumulation in the corrugations and the acoustic

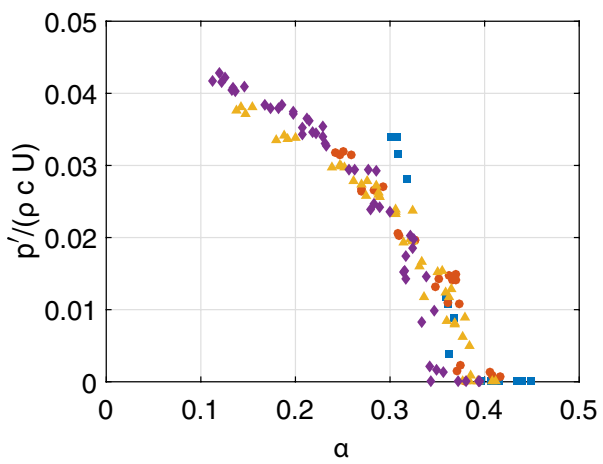

Fig. 15 Dimensionless acoustic whistling amplitude $\left(p^{\prime} /(\rho c U)\right)$ as a function of rib fill fraction $\alpha$. Symbols are as defined in Fig. 14

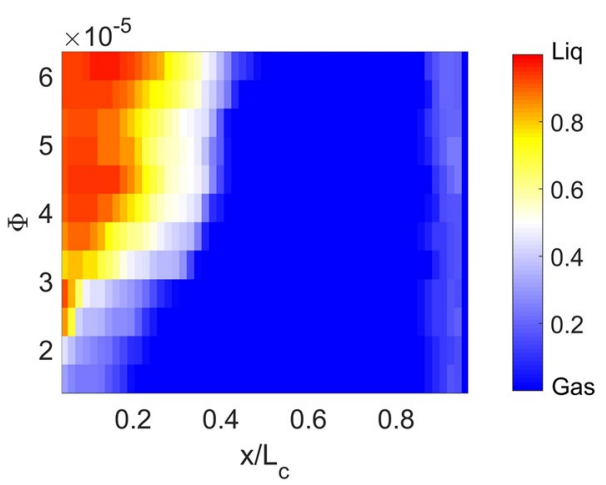

Fig. 16 Development of filling plotted over the cavity length for different liquid addition rates at $M a=0.122\left(U_{\mathrm{b}}=42 \mathrm{~m} / \mathrm{s}\right)$. The color indicates the part of the corrugation depth filled up by liquid at a certain streamwise position along the cavity. Gas flow is from left to right

output shows that the filling behavior is of great importance for whistling mitigation in corrugated pipe flow with liquid addition.

A drawback of using the filling parameter is that it is related to the corrugation geometry. Furthermore, it does not give any insight in the distribution of liquid within the corrugations, and in the way, corrugations are filled. To obtain further insight in the filling behavior, the depthwise filling of the corrugations along the streamwise coordinate is plotted in Fig. 16. It reveals that liquid mainly accumulates at the upstream side of the cavities. It also shows the strong growth in filling at the lower $\Phi_{1}$ range, which stabilizes at higher liquid injection rates. The filling at the downstream bottom corner of the ribs is very stable and less sensitive to changes in the incoming liquid content of the flow. A further increase of the filling there is limited by the impinging gas jet that is directed towards the cavity bottom from the stagnation point at the aft corrugation edge.

A corrected cavity length was used to further generalize the results and compare between the different cases. This 


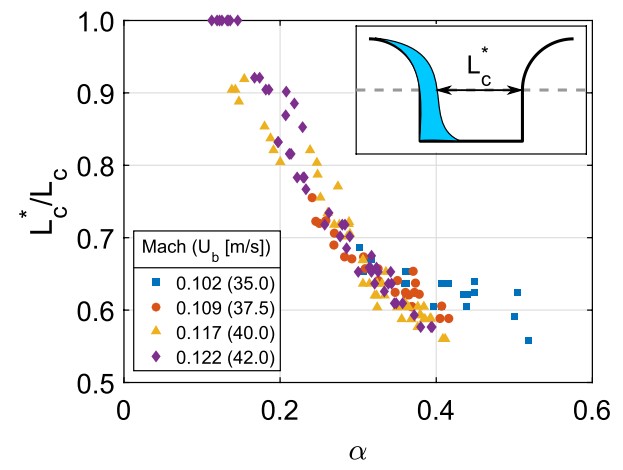

Fig. 17 Corrected cavity length $L_{\mathrm{c}}^{*}$, sampled at half cavity depth versus rib fill fraction $\alpha$. The insert shows the definition of $L_{\mathrm{c}}^{*}$

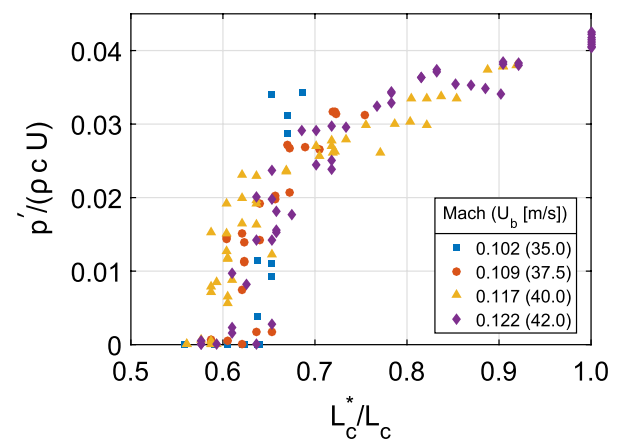

Fig. 18 Dimensionless acoustic whistling amplitude $p^{\prime} /(\rho c U)$ relative to the corrected cavity length $L_{\mathrm{c}}^{*}$

length is found by identifying the space that is not occupied by liquid within the cavity for at least $50 \%$ of the time. The streamwise length of this space at $0.5 H_{c}$ is plotted against $\alpha$ in Fig. 17. The overlap between the plotted data indicates a close resemblance of the filling profiles at different gas flow speeds. The region with $0.6 \leq L_{\mathrm{c}}^{*} / L \leq 0.7$ seems to be critical, since the whistling amplitude strongly decreases in this region (see Fig. 18). The critical behavior could be related to the effective length of the cavity, dropping below the whistling threshold governed by the cavity length over the momentum thickness of the incoming boundary layer $\left(L_{\mathrm{c}}^{*} / \theta\right.$; see Gharib and Roshko 1987). Also the changed upstream rib geometry might be causing the reduction in noise production. The alteration of the rib geometry by liquid flowing over the rib can directly change the shear layer dynamics. However, it can also change the acoustics. Nakiboğlu et al. (2010) showed that an alteration of the upstream rib can have a significant effect on the whistling. As the rib edge becomes sharper $\left(r_{\text {ed }}\right.$ goes down), an increased sound absorption takes place due to a local increase of the wall-normal acoustic velocity there, yielding a reduction in acoustic source strength.

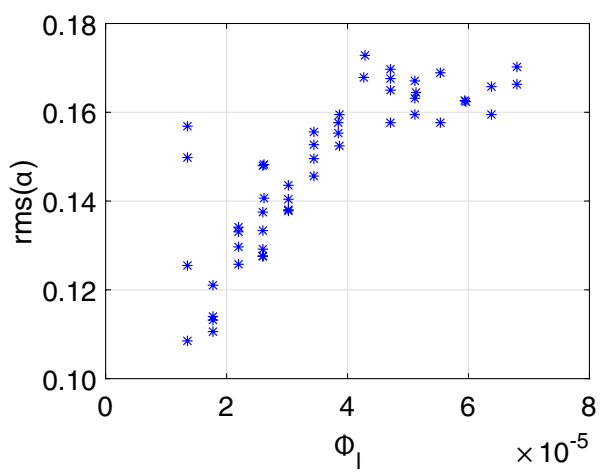

Fig. 19 Root-mean-square values of the spatial variation in filling ratio $\alpha$ over the ribs in view as a function of the liquid addition rate and for $M a=0.122\left(U_{\mathrm{b}}=42 \mathrm{~m} / \mathrm{s}\right)$

Apart from the filling itself, temporal and spatial variations of the liquid accumulation in the individual ribs might be another factor reducing the whistling in these pipes, effectively breaking the axial periodicity of the corrugations. Figure 19 shows the root-mean-square (rms) values of the filling variations in space as a function of liquid injection rate. A slight increase of variations in $\alpha$ with increasing $\phi_{1}$ is observed, but it is rather limited when compared to the accompanying increase in $\alpha$ from 0.1 to 0.4 . The fill fraction itself, therefore, seems to be more important for the whistling behavior of the corrugated pipe than the variability in the filling.

\subsubsection{Downward gas flow}

The filling pattern of the corrugations along the pipe was demonstrated to be essential for upward directed flow. To modify the typical filling behavior, the setup was flipped upside-down, effectively changing the direction of the gravity vector and thereby changing the filling profile of the corrugations. The gas flow is now in the downward direction and has the same orientation as the gravity vector.

The whistling behavior of the corrugated pipe without liquid injection was very similar to the upward oriented gas flow results, small differences in whistling amplitudes could be explained from differences in piping length between the two cases.

When liquid was injected to the incoming gas flow, whistling was eliminated completely, even for the lowest liquid fractions. The onset velocity increased to a value above $U=42 \mathrm{~m} / \mathrm{s}(M a=0.122)$, which is the maximum that could be reached with the current setup. PLIF imaging of the individual rib filling showed a substantial increase in filling compared to the upward oriented gas flow case, for the same incoming liquid fraction. A typical filling profile of a cavity in the downward experiments is shown 


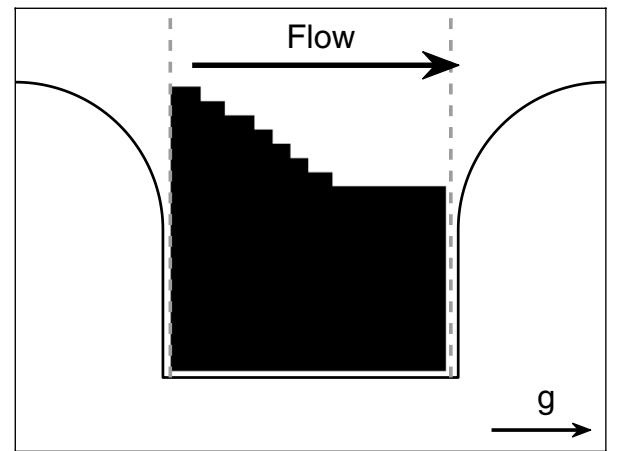

Fig. 20 Averaged rib filling for downward oriented gas flow. Flow is from left to right, $M a=0.117(U=40 \mathrm{~m} / \mathrm{s})$ and $\phi_{\mathrm{L}}=1.85 \times 10^{-5}$ $\left(Q_{1}=85 \mathrm{~mL} / \mathrm{min}\right)$. Dark regions are regions of liquid accumulation and the area outside dashed line has been masked

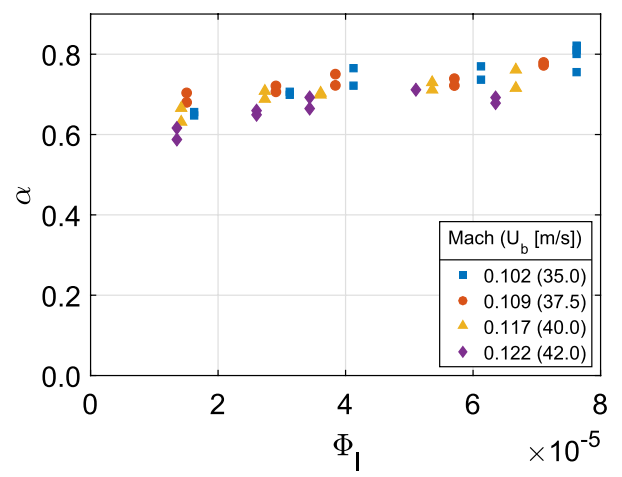

Fig. 21 Fill fraction in the downward experiments for different liquid volume fractions in the incoming flow. Compare with Fig. 14 for upward gas flow

in Fig. 20. As in the upward directed case, liquid tends to accumulate close to the upstream cavity wall. However, in this case, almost the entire rib is filled with liquid. For all cases, filling increases to values above $60 \%$ of the corrugation volume (Fig. 21). The cavities, therefore, experience a filling that is always higher than the whistling threshold, found in the upward directed gas flow experiments. Furthermore, an increase in the liquid volume fraction causes only a slight increase in liquid accumulating in the corrugations, and the trend is comparable for different gas flow speeds. This reveals that the liquid filling in these flow conditions is more stable, reaching an equilibrium that is almost insensitive to $\Phi_{1}$.

\section{Discussion}

Several mechanisms behind the whistling mitigation by liquid addition were proposed by Belfroid et al. (2013). Here, we recapitulate these proposed mechanisms: (1) filling of corrugations with liquid, reducing the depth of the cavities; (2) additional acoustic damping due to the (dispersed and annular) two-phase flow; and (3) a reduced acoustic source strength due to an increased boundary layer thickness. Based on the findings, in the present work, it appears that mitigation of whistling is related predominantly to the filling of the cavities. There is a strong correlation between the filled geometry and the acoustic output, for different free stream velocities. The first mechanism, therefore, seems to be dominant. Reduction of the whistling amplitude can then be related to a reduction of cavity size, a changed geometry of the upstream cavity edge, or a reduction of the cavity length, resulting in a sub-critical $L_{\mathrm{c}} / \theta$ ratio where no whistling occurs anymore.

Although, in many applications, corrugated pipes are applied in upward flow direction, downward experiments were also carried out to confirm the importance of cavity filling when it comes to a reduction of sound production. The differences between upward and downward experiments can be interpreted from the forces influencing the liquid accumulation within the corrugations. These forces are described in Sect. 1.2. The main difference between downward and upward flow is the direction of the gravity vector with respect to the flow direction. The Bond and Weber number ranges, presented in Table 1, show the significance of gravity in the studied experimental conditions. In downward orientation, gravity is aligned with the flow direction, whereas in upward orientation, it is against the flow direction. This causes the gravitational force to prevent emptying of the corrugations in the downward flow case, whereas it promotes emptying of the corrugations in the upward flow case, leading to the different filling behavior observed in the experiments.

\section{Conclusions and outlook}

The present study provides more insight in the mechanisms behind the mitigation of whistling in corrugated pipes by means of liquid addition. This was achieved by an experimental investigation into whistling in upward oriented flow through corrugated pipes, using a combination of acoustic measurements to reconstruct the acoustic waves in the pipe and PLIF imaging of the liquid accumulating in the cavities.

The acoustic measurements show that the amplitude of whistling decreased linearly with increasing liquid volume fraction in the incoming flow. The reduction in sound was the strongest for lower gas velocities, resulting in an increase of the onset velocity of whistling to values above $30 \mathrm{~m} / \mathrm{s}$ for the lowest liquid volume fractions. The PLIF 
images were used to identify the amount of liquid accumulating in the corrugations. A filling threshold was found above which no whistling occurred, within the observed range of gas flow velocities. Furthermore, there was a strong correlation between the attained reduction in whistling amplitude and the fill fraction of the corrugations. This indicates that the amount of filling is a very important factor in reducing the sound production from corrugated pipes.

To confirm these findings, the flow direction was reversed (to downward oriented flow). The filling behavior was entirely different when the flow was from top to bottom. A significantly higher filling of the corrugations was exhibited over the entire range of gas flow speeds and liquid fractions added, explaining the absence of whistling in experiments with downward oriented gas flow. The effect of the gas flow speed and liquid volume fraction on the filling was considerably smaller compared to upward oriented flow, indicating a more stable filling behavior. This is caused by the change in direction of the gravity, which in downward flow experiments counteracts the inertial forces, whereas in upward flow experiments, it works together with the inertial forces to empty the corrugations. The higher filling ratio in downward oriented gas flow resulted in an absence of whistling, irrespective of the gas flow rates and liquid injection rates.

Geometrical alterations are likely the main source of whistling mitigation in two-phase flows in corrugated pipes. Rib-to-rib fluctuations do not increase significantly upon increasing the liquid fraction in the inflow and are not expected to be essential for noise reduction. The effective shortening of the cavity length could eventually lead to reduced values of $L / \theta$ of the incoming boundary layers, and eventually below the whistling threshold. Furthermore, a geometric alteration of the upstream cavity edge could also reduce whistling. These two mechanisms are expected to play an important role in noise reduction in corrugated pipe flow by liquid addition. Future work should encompass parametric studies to investigate the effect of the corrugation geometry and liquid properties on whistling mitigation by a liquid phase. In addition, the effect of other possible noise mitigation mechanisms (acoustic damping due to droplets and shear layer disruption) should be studied in more detail. This would give a broader insight in the individual effects of the different mechanisms.

Open Access This article is distributed under the terms of the Creative Commons Attribution 4.0 International License (http:// creativecommons.org/licenses/by/4.0/), which permits unrestricted use, distribution, and reproduction in any medium, provided you give appropriate credit to the original author(s) and the source, provide a link to the Creative Commons license, and indicate if changes were made.

\section{References}

Belfroid S, Golliard J, Vijlbrief O (2013) Singing mitigation in corrugated tubes with liquid injection. In: ASME 2013 Pressure Vessels and Piping Conference, American Society of Mechanical Engineers

Belfroid S, Korst H, van Beek P, Lunde K, Eidsvik I, Hansen F, Olsen B (2014) Singing mitigation in an export riser via liquid injection: a field case study. In: ASME 2014 Pressure Vessels and Piping Conference, American Society of Mechanical Engineers

Binnie A (1961) Self-induced waves in a conduit with corrugated walls ii. Experiments with air in corrugated and finned tubes. Proc R Soc Lond A Math Phys Eng Sci R Soc 262:179-191

Bruggeman J, Hirschberg A, Van Dongen M, Wijnands A, Gorter J (1991) Self-sustained aero-acoustic pulsations in gas transport systems: experimental study of the influence of closed side branches. J Sound Vib 150(3):371-393

Burstyn W (1922) Eine neue pfeife (a new pipe). Z Tech Phys(Leipzig) 3:179-180

Cadwell LH (1994) Singing corrugated pipes revisited. Am J Phys 62(3):224-226

Cermak P (1922) Über die tonbildung bei metallschläuchen mit eingedrücktem spiralgang (on the sound generation in flexible metal hoses with spiraling grooves). Phys Z 23:394-397

Crawford FS (1974) Singing corrugated pipes. Am J Phys 42(4):278-288

Curle N (1955) The influence of solid boundaries upon aerodynamic sound. Proc R Soc Lond A Math Phys Eng Sci R Soc 231:505-514

Dokumaci E (1995) Sound transmission in narrow pipes with superimposed uniform mean flow and acoustic modelling of automobile catalytic converters. J Sound Vib 182(5):799-808

Elliott J (2005) Corrugated pipe flow. Lecture Notes on the Mathematics of acoustics 207-222

Froessling N (1938) Über die verdunstung fallender tropfen. Gerlands Beiträge zur Geophysik 52:170-215

Gharib M (1993) Active control of flow induced resonance in continuous corrugated tubes. Tech. rep, DTIC Document

Gharib M, Roshko A (1987) The effect of flow oscillations on cavity drag. J Fluid Mech 177:501-530

Golliard J, Belfroid S, Vijlbrief O (2013) Acoustic damping in smooth and corrugated pipes with and without liquid injection. In: ASME 2013 Pressure Vessels and Piping Conference, American Society of Mechanical Engineers

Goyder H (2010) On the modelling of noise generation in corrugated pipes. J Press Vessel Technol 132(4):041304

Häber T, Gebretsadik M, Bockhorn H, Zarzalis N (2015) The effect of total reflection in plif imaging of annular thin films. Int J Multiph Flow 76:64-72

Howe MS (2003) Theory of vortex sound. Cambridge University Press, Cambridge, UK

Jang SH, Ih JG (1998) On the multiple microphone method for measuring in-duct acoustic properties in the presence of mean flow. $\mathrm{J}$ Acoust Soc Am 103(3):1520-1526

Kieffer SW (1977) Sound speed in liquid-gas mixtures: water-air and water-steam. J Geophys Res 82(20):2895-2904

Koschatzky V, Westerweel J, Boersma BJ, Scarano F, Moore PD (2011) High speed PIV applied to aerodynamic noise investigation. Exp Fluids 50(4):863-876

Leutheusser HJ (1964) Flow nozzles with zero beta ratio. J Basic Eng 86(3):538-540

Levine H, Schwinger J (1948) On the radiation of sound from an unflanged circular pipe. Phys Rev 73(4):383-406

Nakiboğlu G, Belfroid SPC, Willems JFH, Hirschberg A (2010) Whistling behavior of periodic systems: corrugated pipes and multiple side branch system. Int J Mech Sci 52(11):1458-1470 
Nakiboğlu G, Belfroid S, Golliard J, Hirschberg A (2011) On the whistling of corrugated pipes: effect of pipe length and flow profile. J Fluid Mech 672:78-108

Nakiboğlu G, Manders H, Hirschberg A (2012a) Aeroacoustic power generated by a compact axisymmetric cavity: prediction of selfsustained oscillation and influence of the depth. J Fluid Mech 703:163-191

Nakiboğlu G, Rudenko O, Hirschberg A (2012b) Aeroacoustics of the swinging corrugated tube: voice of the dragon. J Acoust Soc Am 131(1):749-765

Peerlings L (2015) Methods and techniques for precise and accurate in-duct aero-acoustic measurements: application to the area expansion. PhD thesis, KTH Royal Institute of Technology

Petrie A, Huntley I (1980) The acoustic output produced by a steady airflow through a corrugated duct. J Sound Vib 70(1):1-9
Rajavel B, Prasad MG (2013) Acoustics of corrugated pipes: a review. Appl Mech Rev 65(5):050801

Ronneberger D, Ahrens C (1977) Wall shear stress caused by small amplitude perturbations of turbulent boundary-layer flow: an experimental investigation. J Fluid Mech 83(03):433-464

Rudenko O, Nakiboğlu G, Holten A, Hirschberg A (2013) On whistling of pipes with a corrugated segment: experiment and theory. J Sound Vib 332(26):7226-7242

Sanna F, Golliard J, Belfroid S (2015) On the effect of water film on flow-induced pulsations in closed side branches in tandem configuration. In: ASME 2015 Pressure Vessels and Piping Conference, American Society of Mechanical Engineers

Weng C (2015) Theoretical and numerical studies of sound propagation in low-mach-number duct flows. PhD thesis, KTH Royal Institute of Technology 